\title{
Rhinocerebral Mycosis: Combined Modality Protocol
}

\author{
${ }^{1}$ Ashok K Gupta, ${ }^{2}$ Gopika Kalsotra \\ ${ }^{1}$ Professor and Head, Department of Otolaryngology and Head and Neck Surgery (Unit-II), Postgraduate Institute of Medical \\ Education and Research, Chandigarh, India \\ ${ }^{2}$ Senior Resident, Department of Otolaryngology and Head and Neck Surgery (Unit-II), Postgraduate Institute of Medical Education \\ and Research, Chandigarh, India
}

Correspondence: Ashok K Gupta, Professor and Head, Department of Otolaryngology and Head and Neck Surgery (Unit-II) Postgraduate Institute of Medical Education and Research, Chandigarh, India

\begin{abstract}
Rhinocerebral mycosis is an invasive fungal infection which occurs primarily in the paranasal sinuses and progresses to involve the brain and/or the orbit. It is commonly seen in immunocompromised individuals and can be most effectively treated if diagnosed early, when it is limited to the nasal cavity and the paranasal sinuses. Even though it is acceptable that surgical debridement in combination with antifungal therapy can lead to high rate of cure, the surgical approach for intracranial extension is still a subject of debate. Twenty-four cases of rhinocerebral mycosis managed at our institute are discussed, regarding presenting complaints and management.
\end{abstract}

Keywords: Rhinocerebral mycosis, itraconazole, Amphotericin B, mucormycoses, invasive.

\section{INTRODUCTION}

Rhinocerebral mycosis is a rapidly progressive fatal opportunistic infection, predominantly affecting people in an immunocompromised state. Paltauf ${ }^{1}$ was first to identify a case of rhinocerebral mycosis in 1885. Aspergillosis and zygomycosis are predominantly responsible for CNS mycosis. Candida, coccidiodes, histoplasma and cladosporium are the other fungi causing CNS mycosis. ${ }^{2}$ In rhinocerebral mycosis the disease originates in the nasal/ sinus mucosa, after inhalation of fungal spores and takes a rapidly progressive course by extending to neighboring tissues including the orbit and central nervous system. ${ }^{3}$ Intracranial extension from paranasal sinuses may occur through destruction of posterior table of frontal sinus, transphenoid route or rhino-orbito-cerebral route. Intracranial extension of paranasal sinus mycosis is a difficult problem to manage. Even though it is acceptable that surgical debridement in combination with antifungal therapy can lead to high rate of cure, the surgical approach for intracranial extension is still a subject of debate. Twenty four cases of rhinocerebral mycosis managed at our institute are discussed, regarding presenting complaints and management.

\section{MATERIAL AND METHODS}

A prospective study was conducted in the Department of Otolaryngology, Postgraduate Institute of Medical Education and Research, Chandigarh, India.

The study included twenty-four patients of paranasal sinus mycoses with proven intracranial extension between January 2003 and December 2008. A detailed history of nasal obstruction, nasal discharge, visual disturbances, headache, vomiting, fever or any symptom suggestive of focal neurological deficit was taken. History pertaining to diabetes and immune status was also recorded.

A thorough clinical examination was carried out in all the cases including examination of nose and paranasal sinuses, an ophthalmic examination and a neurological examination. Radiological examination included computerized tomography (CT scan) $5 \mathrm{~mm}$ axial and coronal sections and gadolinium enhanced magnetic resonance imaging (MRI). CT scans were done to determine the extent of involvement of nose and paranasal sinuses, base of skull, orbit, bony erosion, soft tissue extension as well as intracranial extension. Gadolinium enhanced magnetic resonance imaging (MRI) was obtained in fifteen patients only.

The diagnosed patients were taken up for surgery. In eighteen cases an endoscopic approach was used. Difficulty 
was encountered in removing the disease from the orbital apex, superior to sphenoid sinus and also when the defect in skull base was small and the disease was extensive. In six patients disease was removed by anterior craniofacial resection technique by two teams of ENT-head and neck surgeons and neurosurgeons working simultaneously. In these patients there was a smaller defect in skull base with extensive intracranial extension of the disease or there was frank involvement of frontal lobe.

The operative specimens were sent for mycological and histopathological examination. The mycological studies included direct microscopy in $10 \% \mathrm{KOH}$ to demonstrate fungal elements. All specimens were cultured in Sabouraud glucose agar medium for growth of fungus. Histopathological examination also included special staining such as PAS wherever there was difficulty in diagnosis.

All patients with evidence of tissue invasion were treated with antifungal drugs such as amphotericin, itraconazole and voriconazole. Postoperatively patients were subjected to regular saline irrigations, steroid sprays, low dose systemic steroids and regular nasal endoscopic examination with suction clearance. All the patients have been followed up to the present time for recurrence of symptoms and signs in order to know the effectiveness of each surgical procedure combined with antifungal therapy.

\section{RESULTS}

Twenty four patients suffering from paranasal sinus mycoses with intracranial extension were included in this study. These included cases of mucormycoses ${ }^{5}$ and aspergillosis. ${ }^{19}$ Of these 19 cases of aspergillosis, fourteen were invasive aspergillosis and five were noninvasive aspergillosis.

\section{PREDISPOSING FACTORS}

Predisposing factors included diabetes mellitus (16.7\%), immunosuppressive drugs (12.5\%) and post-transplant (4.2\%).

\section{CLINICAL PRESENTATION}

The age range was $16-52$ years (mean 32.6 years). The major presenting symptoms were nasal discharge (75\%), nasal obstruction (75\%), headache (66.7\%), proptosis (41.7\%), diminution of vision (37.5\%), retro-orbital pain (20.8\%), diplopia (16.7\%) and seizures (4.2\%). History of previous surgery was present in 8 of the patients.

\section{RADIOLOGICAL STUDIES}

All patients underwent noncontrast CT scans, while MRI was performed in 15 patients. CT scan revealed heterogeneous soft tissue density mass with occasional calcifications in different sinuses. Ethmoids were the most commonly involved (83.3\%). Maxillary sinus was involved in 18 cases (75\%). The sphenoid sinus was found to be involved in 14 cases (58.3\%). Frontal sinus was involved in 13 cases (54.1\%). In 10 patients (41.6\%), intraorbital extension was seen (Fig. 1). Subcutaneous extension was noted in 4 cases. MRI was helpful for better delineation of the extent of the disease (Fig. 2). Intracranial extension was seen in the form of destruction of posterior table of frontal sinus (Fig. 3), sphenoid sinus roof (Fig. 4), sphenoid sinus lateral wall (Fig. 5) and rhino-orbito-cerebral. The incidence of route of intracranial extension is shown in Figure 6.

\section{SURGICAL MANAGEMENT}

In eighteen cases an endoscopic approach was used. Difficulty was encountered in removing the disease from the orbital apex, superior to sphenoid sinus and also when the defect in skull base was small and the disease was extensive. In six patients disease was removed by anterior craniofacial resection. These were the patients with small defect in skull base was small with extensive disease, and frank involvement of frontal lobe.

Multiple polyps and brownish or grayish black cheesy material was found in cases with aspergillosis whereas black

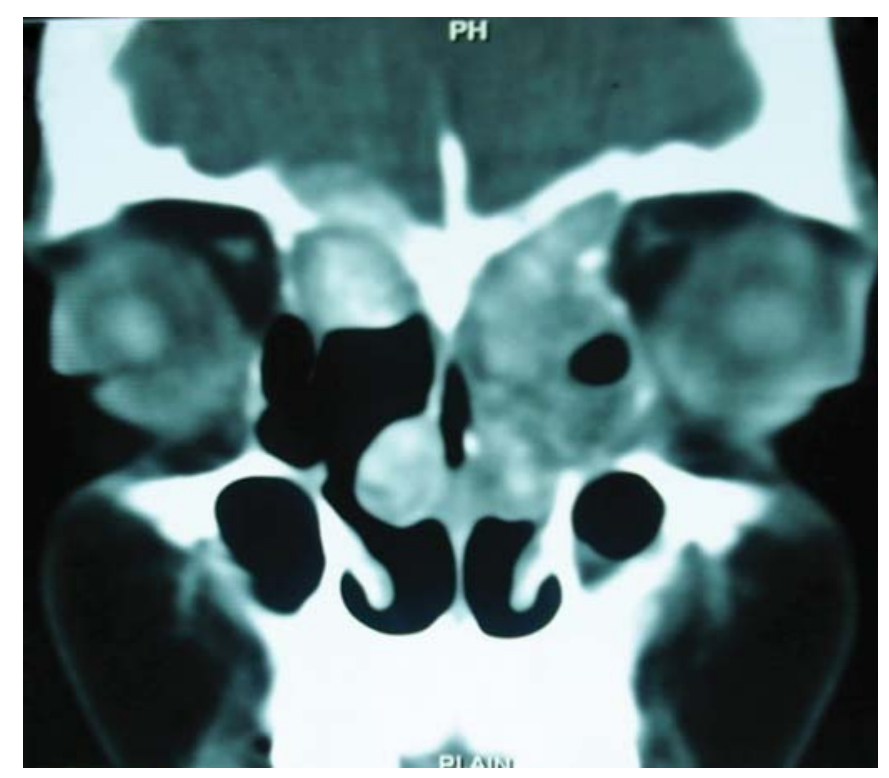

FIGURE 1: Computed tomography of nose and PNS showing heterogeneous opacity on the right side with intracranial extension and of the left side involving frontal sinus and the orbit 


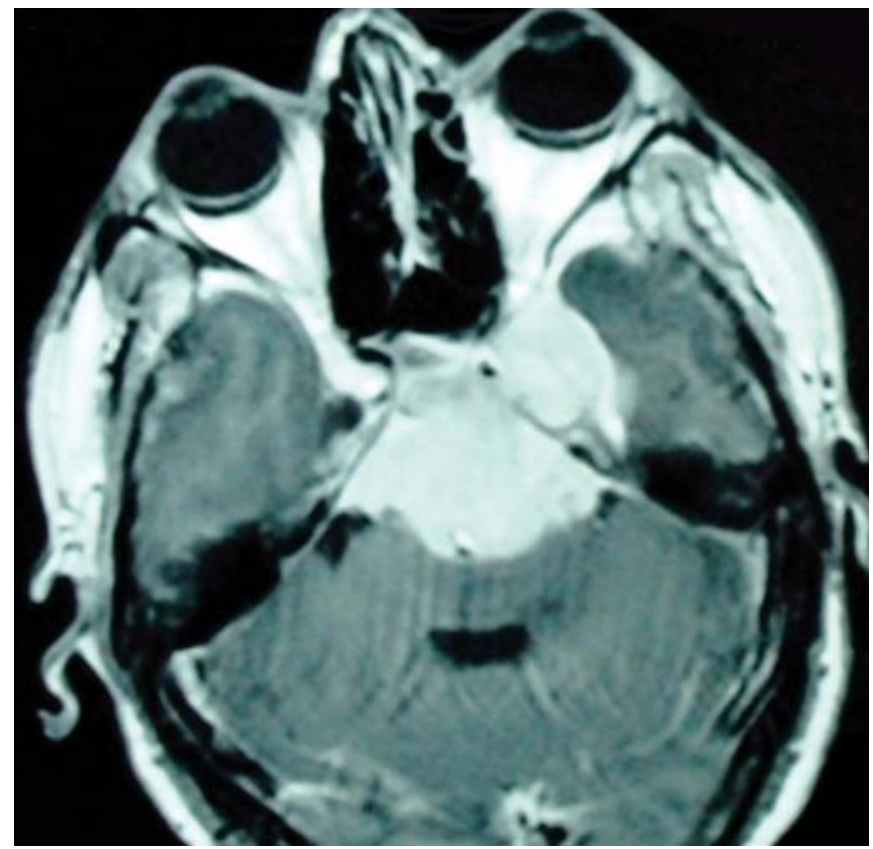

FIGURE 2: Magnetic resonance imaging (T1 weight images) showing extensive intracranial extension

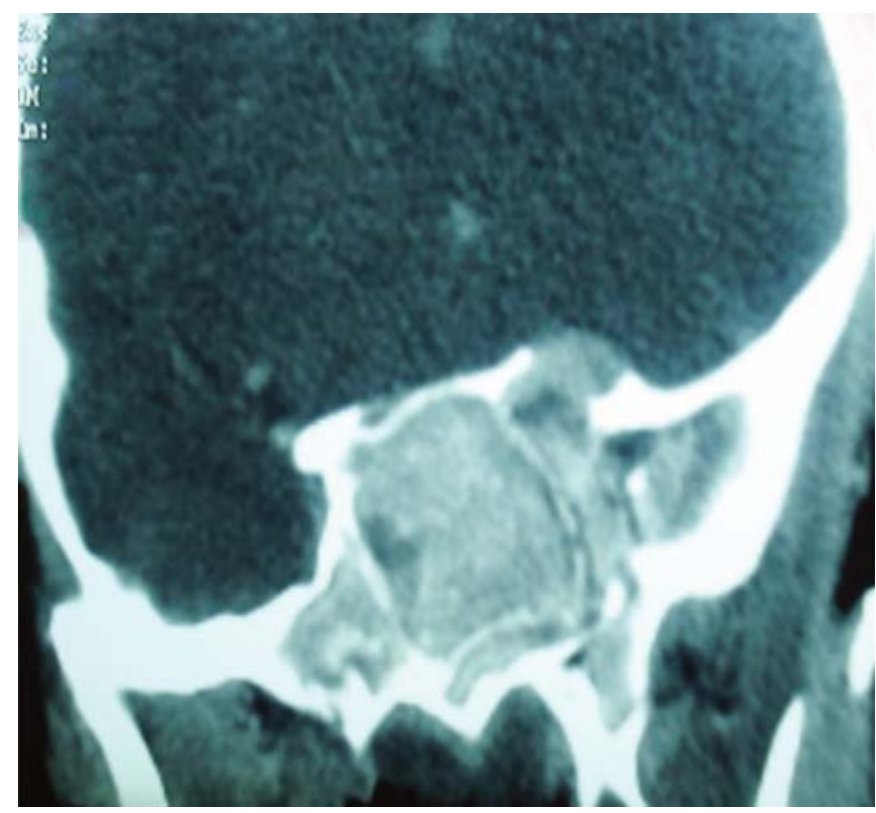

FIGURE 4: Computed tomography of nose and PNS showing heterogeneous opacity in the sphenoid sinus with destruction of lateral wall and roof of sphenoid sinus with destruction of optic tubercle

necrotic material was noticed in cases with mucormycosis. During surgery, the ethmoid sinuses were found to be involved in all the cases. The maxillary sinus was involved in 15 cases (62.5\%) and 12 cases (50\%) had sphenoid sinus involvement, compared with preoperative CT scan which showed 58.3\% involvement. This disparity was due to

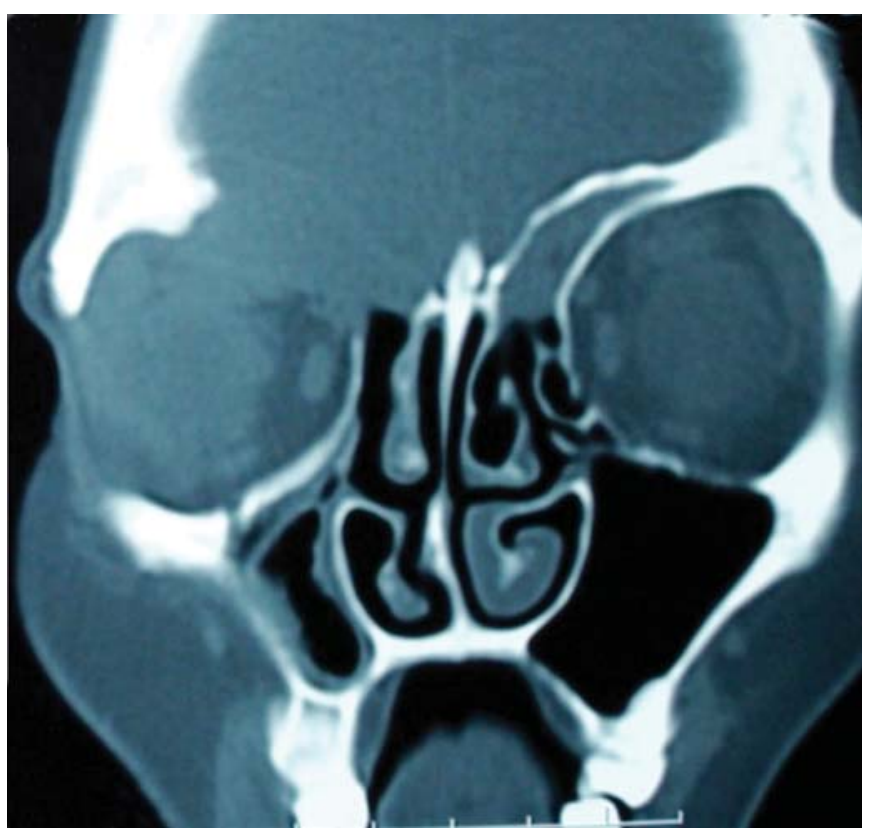

FIGURE 3: Computed tomography of nose and PNS showing destruction of the posterior table of frontal sinus and intraorbital extension

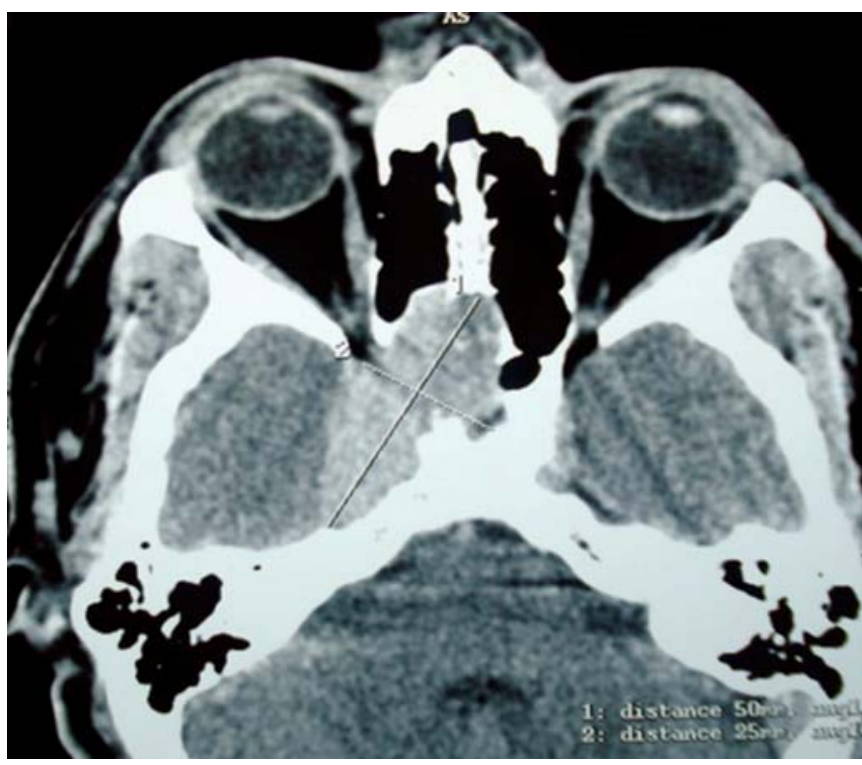

FIGURE 5: Computed tomography of nose and PNS showing destruction of lateral wall of sphenoid sinus with middle cranial fossa involvement

retained secretions in the sinuses. Frontal sinus was found to be involved in 13 cases (54.1\%). The orbit was found to be involved in 9 cases (37.5\%). In one case, the disparity between operative and radiological findings was present. The intraoperative fungal smear was positive in 15 cases of aspergillus. None of the smears were mucor positive, but 


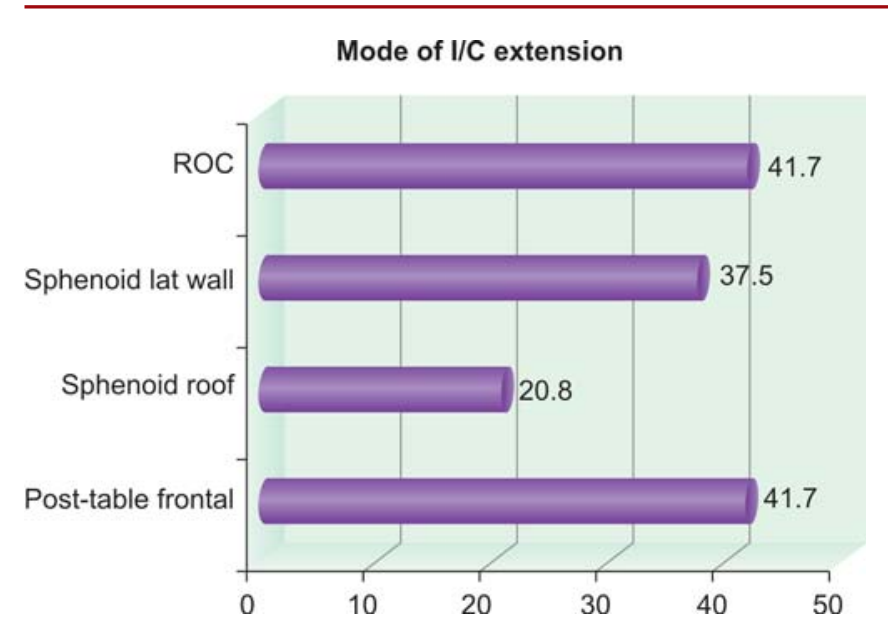

FIGURE 6: Route of intracranial spread of fungus

all cases were culture positive for fungi. Aspergillus flavus was cultured in 17 cases (70.83\%), A. fumigatus and A. niger were positive in one case each $(4.16 \%)$ and mucor was cultured in five cases (20.83\%). Three patients required multiple debridements.

\section{POSTOPERATIVE COMPLICATIONS}

Two patients (9\%) that were treated with endoscopic approach developed CSF rhinorrhea. Primary repair was performed in all cases. None of the other patients developed any significant complication.

\section{HISTOPATHOLOGIC EXAMINATION}

Tissue invasion was noted in fourteen cases of aspergillosis and five cases of mucormycosis. The rest of the tissues were found not to be invaded by fungus.

\section{POSTOPERATIVE ANTIFUNGAL THERAPY AND FOLLOW-UP}

After surgical debridement, all the patients with mucormycosis were administered amphotericin $B$, in a dose of $1 \mathrm{mg} / \mathrm{kg}$ body weight to the total dose of 2.5-4.0 gm.

The onset of nephrotoxicity, was the usual cause for limiting the dose of amphotericin B. Liposomal amphotericin B was used in only one of five patients due to cost constraints.

In cases of invasive aspergillosis, half of the patients were administered itraconazole (200 mg twice daily for six months) and half amphotericin B. In case of no improvement after one month of itraconazole therapy (30\%), amphotericin B was administered.
The patients were followed up for a period varying from 6 to 36 months and a CT scan was repeated after one month of surgery. Six patients had recurrence after six months. In those patients whom had received itraconazole, amphotericin B was administered. In other patients, further dose of amphotericin B was administered. Those patients who were affording $(n=3)$ were given Voriconazole therapy. On the whole, six patients had complete cure of the disease. In fourteen patients the disease was under control. One patient was lost to follow-up. Three patients died; two while on therapy and one due to unrelated cause one month after discharge.

\section{DISCUSSION}

Fungi are ubiquitous organisms occurring in soil, air, body orifices, spoilt food, bread and dust. Inoculation occurs by inhalation, when spores reach the nasopharynx and oropharynx. ${ }^{4}$

Aspergillus and zygomycosis are the commonest organisms causing rhinocerebral mycoses. ${ }^{5,6}$ In the present study aspergillus was found in $79.17 \%$ and Zygomycosis in $20.83 \%$. Micheli was first to describe aspergillus. ${ }^{7}$ Aspergillus is the most common fungus in histologically verified CNS mycosis from India and presents with focal neurological signs and symptoms. ${ }^{8,9}$ Only eight species out of 300 species of aspergillus are authentically involved in human infections. ${ }^{10}$ Nasal and paranasal sinus aspergillosis has been classically divided into (1) allergic aspergillosis; (2) noninvasive; (3) invasive; and (4) fulminant types. ${ }^{1}$

The first report of mucor causing infection human beings was attributed to Paltauf in 1851. Mucormycosis preferentially invades the walls of the blood vessels resulting in vascular occlusion, thrombosis and infarction and even hematogenous dissemination to central nervous system from the primary pulmonary focus. ${ }^{11}$ Vascular occlusion may occur in ophthalmic artery, internal carotid artery and cavernous sinus. ${ }^{11}$

Diabetes mellitus is a common predisposing for CNS mycosis. ${ }^{5,12}$ In our series diabetes was noted in $16.7 \%$ cases. Postrenal transplant state, immunosuppressive therapy and retrovirus infection are other predisposing factors. ${ }^{5,13}$

The commonest manifestations of paranasal sinus mycoses are nasal obstruction and nasal discharge. In our study $75 \%$ patients had nasal discharge and nasal obstruction. $41.7 \%$ patients had proptosis, $37.5 \%$ had defective vision and $16.7 \%$ had ophthalmoplegia of varying 
degrees. Intracranial extension is sometimes associated with headache $(66.7 \%$ in our study). Progressive intracranial extension may lead to lethargy, focal neurological deficit, coma and seizures (one case). Some may present with subcutaneous extension of disease (four cases in this series). Aggressive infection of the orbit with intracranial extension may manifest with various cranial nerve palsies. Cavernous sinus invasion with internal carotid artery thrombosis is a common complication, associated with high mortality. ${ }^{14}$

Preoperative contrast-enhanced CT scan is useful in defining the extent of the disease and for treatment planning. ${ }^{15}$ In our series, the CT scan was found to be very sensitive in diagnosing the fungus because all cases with radiological suspicion of fungus were confirmed by histopathology giving an overall sensitivity of $100 \%$ whereas the overall specificity in terms of extension of disease was $78 \%$ in which preoperative CT was compared with operative findings.

MRI is useful in identifying the intradural and intracranial extent of the disease, cavernous sinus thrombosis or thrombosis of cavernous portion of the internal carotid artery. ${ }^{16}$ Perineural spread of the disease can also be demonstrated with contrast-enhanced MRI scans. ${ }^{17}$ MRI was found to be superior in delineating the intracranial spread and in identifying invasion of cavernous sinus by the disease as well as in the detection of vascular complication like ischemia.

Aggressive therapy with antifungal drugs, rigorous glucose control and surgical debridement will improve survival in these patients. ${ }^{5,6,18}$ Surgery for the intracranial extension of mycosis is a formidable task to deal with as it necessitates surgical clearance of the skull base and involved sinuses. The extent of surgical excision should balance the degree of morbidity and mutilation against the life threatening risk, this fungal infection may represent. ${ }^{4}$ Endoscopic sinus surgery may be of help in patients with limited disease and is associated with less morbidity. ${ }^{19}$

Anterior craniofacial resection combined with extracranial approach has been employed earlier when dealing with fulminant aspergillosis. ${ }^{20,21}$ This affords an excellent approach to the involved area and gives direct access to difficult areas such as the orbital apex without jeopardizing vital structures. The dissection is done under vision, so there is very little chance of having residual disease.

Finn $^{22}$ described craniofacial resection along with pansinusectomy and orbital exenteration forintracranial extension of mucormycoses along with chemotherapy.
Abedi et al (1985), ${ }^{23}$ while describing their 25 years experience in treating cerebrorhino-orbital mucormycosis, mentioned the utility of craniofacial resection in three of their patients who had extension to anterior cranial fossa. All three have survived.

Aggressive antifungal therapy is required in these patients. Liposomal amphotericin, administered at the dosage of $5 \mathrm{mg} / \mathrm{kg} /$ daycan build up the drug levels in onefourth of time duration when compared with conventional amphotericin therapy, with minimal renal toxicity and greater tissue penetration..$^{20}$ Cost remains the major limiting factor. In our series, liposomal amphotericin was given to one pastient. The sinusoidal debridement remains essential, even when orbital preservation has been attempted, with varying success. ${ }^{4,19}$ Voriconazole may be tried in resistant cases of rhinocerebral aspergillosis. ${ }^{24}$ In our series voriconazole was given to three patients who were not responding to amphotericin $\mathrm{B}$. The adjuvant therapies range from local amphotericin irrigation, ${ }^{4,25}$ packing, ${ }^{6}$ hyperbaric oxygen, ${ }^{26}$-fluoro-cytosine 15 and even decompressive craniotomy for malignant occlusive stroke. ${ }^{11}$

The most significant aspect in dealing with such patients is the residual or recurrent disease. This is the reason the patients in this series were kept on strict follow-up. The probable cause of recurrence in these cases include (a) presence of disease in close proximity to inaccessible vital structures which was not dealt with; (b) difficulty in removing the disease through a small defect in skull base; (c) dural invasion; (d) brain invasion. ${ }^{21}$ None of our patients in any of the groups had residual disease. The follow-up ranged from 6 to 36 months. The favorable outcome in our series was $83.33 \%$.

Thus, to conclude combined modality protocol (antifungal therapy and meticulous, extensive surgical

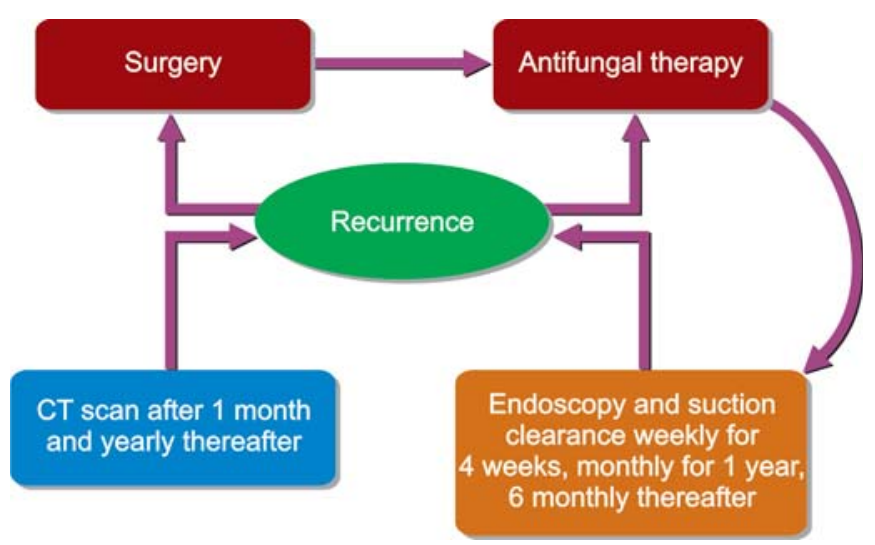

FIGURE 7: Algorithm of management for rhinocerebral mycosis 
debridement ) is required in rhinocerebral mycosis to achieve a good outcome as shown in (Fig. 7). Extensive lesions can be controlled by surgery (endoscopic or external) and antifungal therapy. Regular follow-up is required to look for any signs of recurrent active disease.

\section{REFERENCES}

1. Paltauf A. Mycosis mucorina. Virchows Arch 1885;102:54364.

2. Eucker J, Sezer O, Graf B, Possinger K. Mucormycosis. Mycoses 2001;44:253-60.

3. DeShazo RD, O'Brien M, Chapin K, Soto-Aguilar M, Gardner L, Swain R. A new classification and diagnostic criteria for invasive fungal sinusitis. Archives Otolaryngol Head Neck Surg 1997;123:1181-88.

4. Kohn R, Helper R. Management of limited rhino-orbital mucormycosis without exenteration. Ophthalmology 1985;92:1440-43.

5. Dubey A, Patwardhan RV, Sampath S, Santosh V, Kolluri S, Nanda A. Intracranial fungal granuloma: Analysis of 40 patients and review of literature. Surg Neurol 2005;63:254-60.

6. Nussbaum ES, Hall WA. Rhinocerebral mucormycosis: Changing patterns of disease. Surg Neurol 1994;41:152-56.

7. Micheli PA. Nova Plantarum Genera 1729. Florentiae.

8. Santosh V, Yasha TC, Khanna N. Fungal infections of the nervous system: A pathological study. Neurli Infect Epidemol 1996;1:69-79.

9. Murthy JM, Sundaram C, Prasad VS, Purohit AK, Rammurthi S, Laxmi V. Sinocranial aspergillosis: A form of central nervous system aspergillosis in South India. Mycoses 2001;44:141-45.

10. Rippon WJ. Medical Mycology: The pathogenic fungi and the pathogenic actinomycetes. Aspergillosis (2nd ed) Philadelphia: WB Saunders Co 1982;565-94.

11. Thajeb P, Thajeb T, Dai D. Fatal strokes in patients with rhinoorbito-cerebral mucormycosis and associated vasculopathy. Scand J Infect Dis 2004;36:643-48.

12. Sundaram C, Mahadevan A, Laxmi V, Yasha TC, Santosh V, Murthy JM et al. Cerebral zygomycosis. Mycoses 2005;48:396407.
13. Moraru RA, Grossman ME. Palatal necrosis in an AIDS patient: A case of mucormycosis. Cutis 2000;66:15-18.

14. Quattrocolo G, Pignaatta P, Dimanico U, Tarenzi L, Baglore P. Rhinocerebral mucormycosis and internal carotid artery thrombosis in a previously healthy patient. Acta Neurol Belg 1990;90:20-26.

15. Ochi JW, Harris JP, Feldman JI, Press GA. Rhinocerebral mucormycosis: Results of aggressive surgical debridement and amphotericin B. Laryngoscope 1988;98:1339-42.

16. McDevitt GR Jr, Brantley MJ, Cawthon MA. Rhinocerebral mucormycosis: A case report with magnetic resonance imaging findings. Clin Imaging 1989;13:317-20.

17. McLean FM, Ginsberg LE, Stanton CA. Perineural spread of rhinocerebral mucormycosis. AJNR Am J Neuroradiol 1996;17:114-16.

18. Alfano C, Chiummariello S, Dessy LA, Bistoni G, Seuderi N. Combined mucormycosis and Aspergillosis of rhinocerebral region. In vivo 2006;20:311-15.

19. Avet PP, Kline LB, Sillers MJ. Endoscopic sinus surgery in the management of mucormycosis. J Neuro-ophthalmol 1999;19:5661.

20. Pardal Refoyo JL, Chocarro Martinez A, Brezmes Valdivieso MF, Parr Perez C. Treatment review in rhinocerebral mucormycosis . An Otorrinolaringol Ibero Am 1998;25:45-56.

21. Gupta AK, Mann SBS, Khosla VK, Sastry KVSSRK, Hundal JS. Nonrandomised comparison of surgical modalities for paranasal sinus mycoses with intracranial extension. Mycoses 1999;42:225-30.

22. Finn DG. Mucormycosis of the paranasal sinuses. Ear Nose Throat J 1988;67:813-22.

23. Abedi E, Sismanis A, Choi K, Pastore P. Twenty five years experience treating cerebro-rhino-orbital mucormycosis. Laryngoscope 1984;94:1060-63.

24. Kleinsehmidt-DeMasters BK. Central nervous system Aspergillosis: A 20 years retrospective series. Hum Pathol 2002;33:116-24.

25. Hopkins MA, Treloar DM, Mucormycosis in diabetes. Am J Crit Care 1997;6:363-67.

26. Chassaing N, Valton L, Kany M, et al. Rhinocerebral fungal infection successfully treated with supplementary hyperbaric oxygen therapy . Rev Neurol 2003;159:1178-80. 